\title{
Wer wird morgen noch Ärztin oder Arzt?
}

Die Gesellschaft ist beunruhigt, sie hat Angst und ruft nach Ärzten. Wo sind die Ärzte, die uns im dritten und vierten Alter betreuen werden? Diese medizinischen Leistungserbringer, die Angst nehmen und Sofortgenesung bringen müssen, werden hierzulande in ungenügender Quantität und Qualität bzw. mit unzureichendem Spezialisierungsgrad produziert. Viele Bewerberinnen und Bewerber für das Medizinstudium müssen der begrenzten Aufnahmekapazitäten der medizinischen Fakultäten wegen abgewiesen werden. Die Frage nach den «richtigen» Auswahlkriterien für die limitierten Studienplätze liegt auf der Hand [1].

Der Schweizerische Wissenschafts- und Technologierat (SWTR) empfiehlt in seinem Bericht «Ärztedemographie und Reform der ärztlichen Berufsbildung» [2] eine Erhöhung der Studienplatzkapazität an den medizinischen Fakultäten und eine Reform der ärztlichen Weiterbildung. Letztere setze die bereits erfolgten Reformen des Medizinstudiums fort, und zusammen würden die beiden Massnahmen die medizinische Grundversorgung stärken. Im Bericht werden Ziele und Massnahmen formuliert, ohne vorherige gründliche Analyse der Effizienz des schweizerischen Gesundheitswesens. Das Weiterbestehen der extrem föderalistischen Strukturen, namentlich der kantonalen Spitalstrukturen, wird implizit vorausgesetzt. Es fehlt eine Analyse der Effizienz der medizinischen Fakultäten. Weder die maximale klinische Ausbildungskapazität der Fakultäten noch die Gründe für einen Studienabbruch werden erläutert. Die im europäischen Vergleich hohe Qualität und Effizienz der ärztlichen Weiterbildung in der Schweiz, die Akkreditierung der 44 Weiterbildungsprogramme durch das Eidgenössische Departement des Innern und die breite Abstützung der verantwortlichen Kommission für Weiter- und Fortbildung werden im Bericht schlichtweg skotomisiert.

Die Ärzteschaft steht innerhalb der Leistungserbringer im Gesundheitswesen unter starkem ökonomischem und sozialem Druck: Sie muss ihre Autonomie des Wissens und Handelns gegenüber anderen Leistungserbringern im wahrsten Sinne des Wortes «verkaufen». Die Ärzteschaft sucht nach ihrem Platz in der Arbeitsteilung des Gesundheitswesens - nach dem Platz ihrer Expertise. Was zeichnet eine Ärztin bzw. einen Arzt aus? Welches Berufs- bild, welche Berufsidentität hat er/sie heute? Bereits vor über 50 Jahren hat sich Karl Jaspers in seinem Essay «Die Idee des Arztes und ihre Erneuerung» [3] darüber Gedanken gemacht; eine immer noch spannende und empfehlenswerte Lektüre.

Die Schweizerische Akademie der Medizinischen Wissenschaften (SAMW) wies in einem Bericht auf einen radikalen Wandel des Berufsbildes des Arztes hin. Die Diskussion über das Berufsbild des Arztes, den Professionalismus und die Gesundheitsversorgung wird durch den Bericht der SAMW-Arbeitsgruppe «Die zukünftigen Berufsbilder von Ärztinnen/Ärzten und Pflegenden in der ambulanten und klinischen Praxis» [4] auf eine breitere Basis gestellt. Das Ziel des gesamten Systems wird explizit genannt: die Bedürfnisse der Patientinnen und Patienten. An diesen Bedürfnissen der arbeitsteiligen Gesellschaft sollen sich das Berufsbild und die Aus- und Weiterbildungsziele ausrichten.

Berufsbild, Aus- und Weiterbildung, Berufstätigkeit und Sozialstatus des Arztes werden hinterfragt. Dies ist nicht neu; neu sind die Intensität und die Offenheit, mit denen die Fragen angegangen werden. Dies erlaubt eine frühe aktive Teilnahme an der Gestaltung des angestrebten Berufes. Die heutige Jugend wird - hoffentlich wie frühere Generationen - an der aktiven Mitgestaltung des Arztberufes teilnehmen und sich in ausreichender Anzahl für diesen schönen Beruf, der einzigartige zwischenmenschliche Beziehungen erlaubt, entscheiden.

Dr. med. Max Giger,

Mitglied des Zentralvorstandes der FMH, Präsident Kommission für Weiter- und Fortbildung der FMH

1 Hänsgen K-D. Numerus clausus in der Medizin - werden die Richtigen ausgewählt für Studium und Beruf? Schweiz Ärztezeitung. 2007;88(46):1953-7.

2 www.swtr.ch/d/aktuell_news.html (Zugriff 6. November 2007).

3 Jaspers K. Philosophie und Welt. Reden und Aufsätze. München: Piper; 1958.

4 Arbeitsgruppe «Berufsbilder» der SAMW. Die zukünftigen Berufsbilder von Ärztinnen/Ärzten und Pflegenden in der ambulanten und klinischen Praxis. Schweiz Ärztezeitung. 2007;88(46):1942-52. 